\title{
Monitoring and Analytical Evaluation of Interactivity Mediated by a Dynamic Hypermedia Device
}

\author{
Guillermo L. Rodríguez ${ }^{1}$, Patricia S. San Martín ${ }^{1} \&$ Griselda Guarnieri ${ }^{1}$ \\ ${ }^{1}$ Center International French-Argentine for Systems and Information Sciences (CIFASIS: UNR - CONICET - \\ AMU), Rosario, Argentina \\ Correspondence: Guillermo L. Rodríguez, CIFASIS, CCT-Rosario, Rosario, Santa Fe, CP: 2000, Argentina. Tel: \\ 54-341-4237248. E-mail: guille@ffceia.unr.edu.ar
}

Received: July 12, $2013 \quad$ Accepted: August 24, $2013 \quad$ Online Published: September 11, 2013
doi:10.5539/cis.v6n4p80
URL: http://dx.doi.org/10.5539/cis.v6n4p80

\begin{abstract}
This paper describes the first prototype of an integrated tool that assists people in monitoring and analytical evaluation of interactivity, mediated by a Dynamic Hypermedia Device (DHD). To carry out this practice, the basic technological elements are explained, as well as a model which takes into account experiences related to the addition of new components in implementations solved for this kind of framework. Furthermore, it is also explained how the tool articulates the simulation results obtained from a runtime DEVS (Discrete Event System) that integrates the metrics needed for flexible weights. All the technological developments have been adapted to the educational platform MOODLE as well as to SAKAI collaborative environment. To exemplify this development, a use case has been presented providing functional features and details that outline the methodological steps of the model from the final users' perspective. Thus, an analysis which enables qualitative versatile units in terms of levels of interactivity to the DHD has been achieved.
\end{abstract}

Keywords: Dynamic Hypermedia Device, Interactivity process, social network analysis, information and communication technology

\section{Introduction}

Current Information and Communication Technology (ICT) has enabled the construction of a new physical-virtual reality where people can participate in various socio-technical networks. These networks are comprised in multiple components and relationships, which are configured and reconfigured for the various interactions mediated, depending on a number of requirements.

The need to assess and provide better quality of interactivity processes aims to focus on research and education from responsible participation in physical-virtual contexts. These are especially relevant for the joint construction of the so-called inclusive "Information and Knowledge Society". Taking into account what it is mentioned above, this paper describes the first prototype of an integrated tool called "SEPI-DHD", which assists in the Monitoring and Evaluation Process of Analytical Interactivity mediated by a Dynamic Hypermedia Device (DHD).

A DHD is defined as a heterogeneous network formed by the combination of technologies and social networks, which enable socio-technical subjects of participatory actions -responsible interaction- for management, education, research and cultural production in a workshop environment, exploiting the potential of the ICT (San Martín, Guarnieri, Rodríguez, Bongiovani, \& Sartorio, 2010; Guarnieri, 2010).

In this sense, the DHD is constituted as a complex entity (Gell-Mann, 1995) and consists of the integration of two inseparable dimensions: a technical one (or set of construction techniques that involve a materiality and a particular configuration) and a social one given by the inter-relationships and situations in which they appear.

The need to use SEPI-DHD is based on the importance of evaluative analysis of developments and implementations carried out in educational platforms, collaborative environments and $2.0 \mathrm{Web}$ tools.

To accomplish this, we have adopted the use of DEVS formalism (Discrete EVents Systems) created by Zeigler in 1976. He proposed a theory of discrete event modeling in continuous time systems, allowing a modular description of the phenomena and addressing the complexity using a hierarchical approach. In the implementation of this formalism weighting metrics are integrated following the recommendations of the 
framework INCAMI (Rossi, Pastor, Schwabe, \& Olsina, 2007). In turn, the technological tool has the flexibility of linking the educational platform MOODLE (www.moodle.org) and the collaborative environment SAKAI (www.sakaiproject.org). It also has an original implementation to provide a suitable interface module, responsible for building the pre-conditions and intermediate steps needed to implement the module DEVS (Zeigler, King, \& Praehofer, 2000). What is more, it contains an interface for displaying the results obtained with versatility from different types of filters. In the future, these values may be a new type of contextual information that results from the previous actions of users, and consequently allows an adaptation of collaborative environments or 2.0 Web tools (Sartorio \& Rodríguez, 2010).

It also describes the metrics and the DEVS simulation model, as well as the technological elements necessary for its integration. The use case provides functional features and details related to the methodological steps of the model from the perspective of a final user. Finally, brief conclusions are summarized for the current prospective development.

\section{Metrics and DEVS Models for Simulation}

Measurement and evaluation mechanisms are one of the main activities in the implementation and optimization of the DHD (Rodríguez \& San Martín, 2010).

Measurement processes are critical as they allow quantifying a set of desired characteristics about a specific aspect of a particular entity. This provides a rather detailed view of their status or condition. Meanwhile, the evaluation interprets the values obtained in the measurement. For such processes, measurement and evaluation are necessary to obtain quantitative data from metrics attributes of different entities, and the subsequent interpretation of results from the indicators (Olsina \& Martín, 2004).

There is a good deal of existing information on the definition of metrics and indicators, but no clear consensus on terminology. In this sense, we consider the Ontology Metrics and Indicators presented in Olsina. Molina and Papa (2005) constitute an important proposal for the area of quality management and a valuable contribution to the activities involved in such management. Therefore, this paper will focus on the measurement and evaluation framework called INCAMI (Information Need, Concept model, Attribute, Metric and Indicator) (Rivera, Molina, \& Olsina, 2007).

INCAMI is based on the method WebQEM (Web Quality Evaluation Method) (Olsina \& Rossi, 2002), which in turn is based on models and quality metrics and focuses on the quantitative evaluation of characteristics and attributes of entities. Thus, INCAMI can be used in the design of non-functional requirements, the selection of metrics to quantify the attributes of the entities involved and the interpretation of the corresponding values by indicators.

The first stage of this method corresponds to the definition and specification of the requirements. This module defines the need for information (i.e., the focus of the evaluation) and the design of non-functional requirements, which serve as guides for subsequent activities of measurement and evaluation.

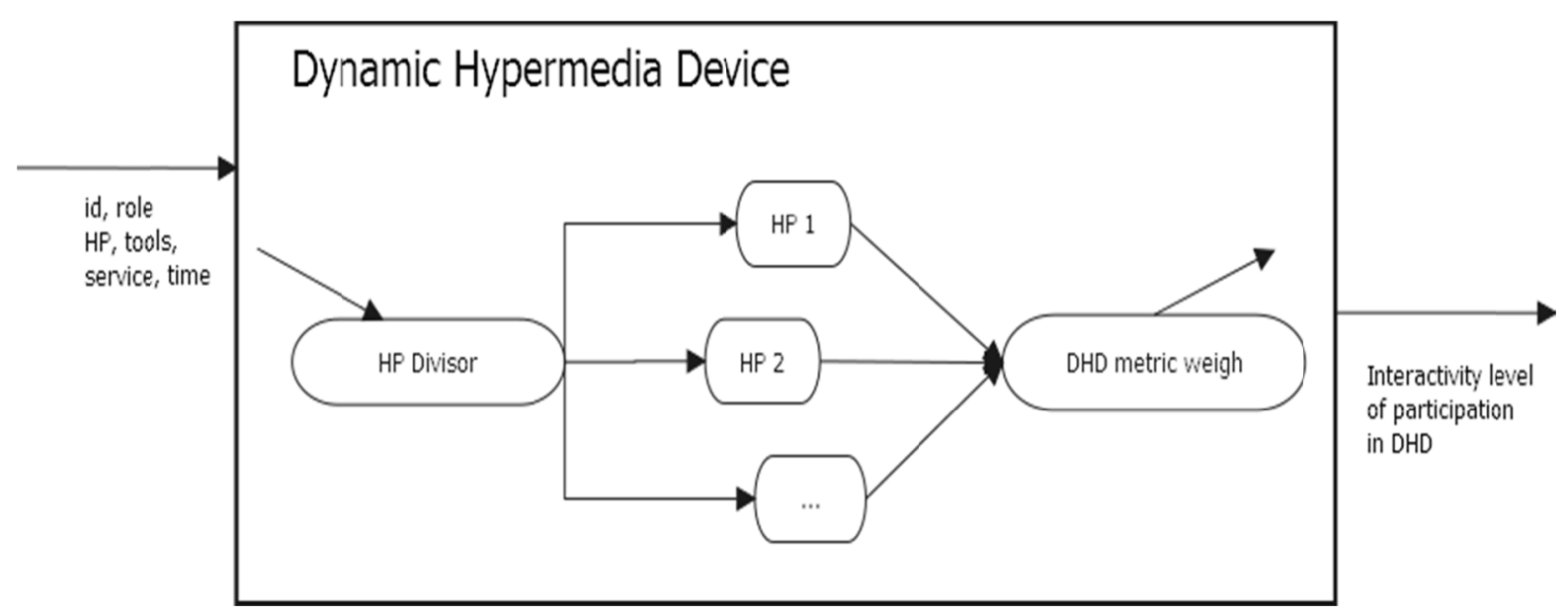

Figure 1. Descriptive model of DEVS coupled modules that integrate a HP 
We take as a starting point the description of the basic conceptual component called Hypermedia Package (HP), where interactions are concretized in DHD. Based on the foundations set out, in line with the technological model presented below, the DEVS model of HP is described in Figure 1.

It is shown that the input event vector is determined by the characteristics of the interaction of the participants, and an output event will result in the level of interactivity for each entry.

If the HP is defined as the basic conceptual components of the DHD, then it must be integrated to obtain the total level of interactivity of each entry.

The complete scheme is shown in Figure 2.



Figure 2. Descriptive model of DEVS coupled modules that integrate a DHD

In this chart, it is shown that the information in our case is a function of the participants' interactions, which are defined by: identification number of the participants (id) and their role; the types of HP and which ones participate; the tools on which to perform the interaction and their type; the service with which it interacts; and the time and date of the interaction.

The second stage is the design and implementation of measurement. This module is focused on defining metrics to be useful for quantifying the attributes, which in the previous step was identified as part of the specification requirements. These are particularly interesting for the project, since the characteristics are measured for the agency to evaluate, considering the need of information provided. That is the ultimate goal of the evaluation, which in our case is the interactivity level of participation.

In both figures, the need to establish three levels of metrics for analysis of interactions in real time is clearly shown, since we can distinguish three different entities: the tool, the weighting of the HP and the weighting of the DHD.

It is essential to understand the construction of the metric, which attributes are measured, and what the partner agencies are. It is also necessary to identify the type of value that is obtained, the drive that is expressed and the type of scale used, in order to allow proper interpretation.

Following the recommendations of the INCAMI model and in order to obtain values for the global indicators, we must consider a model of accumulation and decision criteria. The weighting and accumulation model (clustering) aims at making a well-structured and objective evaluation process, comprehensive for evaluators (or evaluation itself). As in other case studies models, weights and multi-criteria scoring (consensus) were used to designate and set processes. Thus, a defined weighting model (or score), multi-criteria (or consensus) and Logic Preference Scoring (LPS) (Dujmovic \& Bazucan, 1997), in conjunction with synchronization properties, neutrality, adding operations, replacement and other relationships, are based on the mathematical model of weights.

The main objective of the overall assessment implementation is to enable greater levels of flexibility for the values of global and partial indicators. This is achieved by the elementary metric values, which use the model of grouping obtained from the calculation. In this process, these values must be arranged and agreed upon by experts with experience in using such systems. At the same time, it is mentioned that the numerical values indicated only serve as an example of simple application, and education experts of the research team agree with this. The head of the evaluation can add value to the various factors underlining the attribute, which is the most important element in the process. In each case, the resulting value provides a measure of the degree of 
interactivity of participation.

\section{A. Metrics of tool $\mathrm{H}$}

It is built by a product of four factors:

Interactivity level of participation in $\mathrm{H}=\mathrm{Cl} * \mathrm{C2} * \mathrm{C} 3 * \mathrm{C} 4$

These four coefficients should be related to:

Type of tool:

Transmissive format (e.g., links, resources). $C 1=1$

Interactive format (e.g., forums, wiki). $C l=2$

Type of service used:

Create. $C 2=2$

Search. $C 2=1$

Edit. $C 2=2$

Delete. $C 2=1$

Role of the participants:

Teachers. $C 3=1$

Students. $C 3=2$

Users of the tool:

One or two participants. $C 4=1$

Three or more participants. $C 4=2$

B. HP metric weighting

It is built by a product of three factors:

Interactivity level of participation in the $H P=B 1 * B 2 * B 3$

The value of these three factors will be:

Interactivity level of participation in the tool

$B 1=C 1 * C 2 * C 3 * C 4$

Time between the last and current involvement:

If less than one day. $B 2=3$

If less than one week. $B 2=2$

If more than one week. $B 2=1$

Number of tools used:

If three or more tools are used. $B 3=3$

If two tools are used. $B 3=2$

If one tool is used. $B 3=1$

C. DHD metric weighting

The existence of several types of HP (courses and projects in collaborative environments, digital repositories, social networks, etc.), with various features configured both in their tools, and in their related services, show the need to weigh the value obtained from the previous metric to normalize interactivity participation at DHD.

Thus, we obtain:

Interactivity level of participation in the $D H D=A 1 * A 2$

The value of these two coefficients will be: 
Interactivity level of participation in the HP

$A 1=B 1 * B 2 * B 3$

Hypermedia Package Type

If it is a course. $A 2=1$

If it is an electronic document within a Hypermedia Repository. $A 2=2$

Finally, in the evaluation stage, these metrics should be interpreted through indicators to assess or estimate the degree of conformity to the proposed requirements. It is at this point when the indicators should be selected to interpret each metric that quantifies each corresponding attribute in the design of non-functional requirements. The indicators also contain a scale and a function or an algorithm through which it will be possible to interpret the value of the metric, also using a decision rule that will establish thresholds of acceptability of the value obtained.

\section{Technology Integration Model}

The technology integration model determines components, subsystems and relations on the tool SEPI-DHD, which is responsible for articulating the results of the simulation of DEVS model.

Briefly, this tool is handled first by accessing the database and second by applying a transfer function that allows a representation of the values corresponding to the users' interactions. This function generates the correct input values to be used in the simulation. The tool itself contains an appropriate interface for the representation of the coefficients which make up the metrics that are integrated into the DEVS model (Rodríguez, 2010), thereby allowing the charge to control the weights that reflect qualitative properties of the users' interactions.



Figure 3. Integration model for SEPI-DHD

Figure 3 shows the initial connection between the services of the tool and the DEVS simulation model. For this case, the Integrator module methods are represented by a UML class that implements the sequence of executions to be respected. As a result, the DEVS interpreter (in this case PowerDEVS) (Lapadula et al., 2010) take appropriate input values (method get_inputDEVS) that are processed through a parameterized transfer function (method set_Transf). Afterwards, output values are taken (method get_outputDEVS) for further processing. All input data is extracted from the database belonging to the collaborative environment used, through the private method get_DB. The parameters involved in the implementation of the DEVS simulator must be met by the interfaces represented in the Integrator class.

Issues of parameterization and representation of values, pre and post-conditions located at a higher level to achieve better performance and handling by users, are stored in the configuration file and the S.Conf service intervenes.

The integration design presented in this case is based on the description of software components. The integration 
tool proposed here is necessary to consider other elements in this design, which have already been used in this case. In another research (Sartorio \& Cristiá, 2009) each of the above areas were developed in detail.

\section{Use Case Implementation}

We will develop a use case providing functional details on the tool from the perspective of the final user. This path is divided into five methodological steps, which are consistent with the relationships described in Figure 3.

\section{A. Step 1: Access to the database}

This step is transparent to the final user. The designers must meet certain requirements to suit the needs of the integration model proposed here. On the one hand, we study different types of connections between subsystems through messages and the sequence of task performance. On the other hand, it is necessary to implement penetration measures between systems which must comply with a technological infrastructure and design. This is the case of linkage that is proposed in the integration model of the integrator module and database containing interactions. At this point, the environment we are working on must be selected: MOODLE or SAKAI.

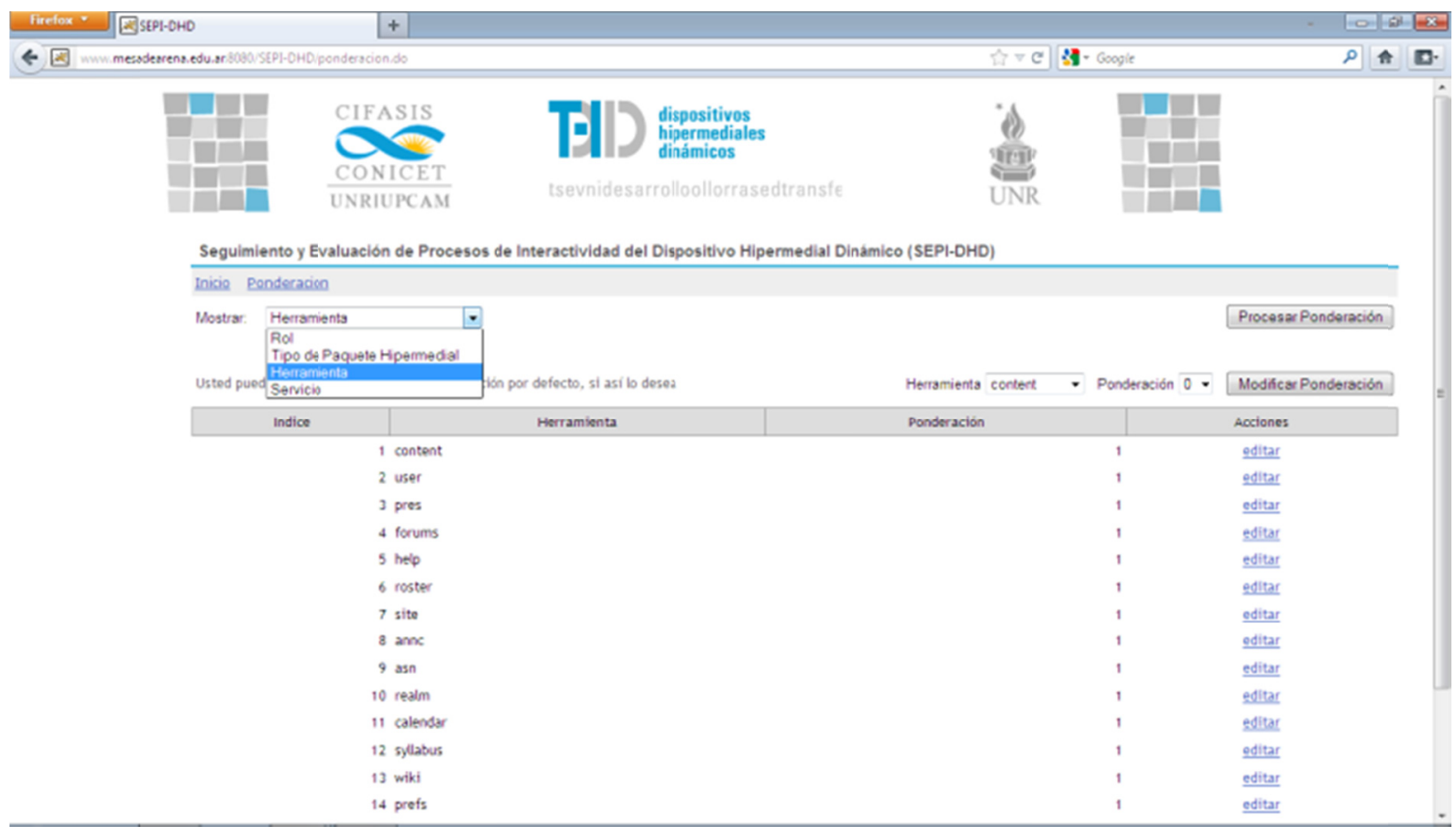

Figure 4. Start implementation of SEPI-DHD

\section{B. Step 2: Coefficients that comprise the metric}

The tool contains a suitable interface for the representation of the coefficients that make up the meter, thus allowing the evaluator to check the weights that reflect qualitative/quantitative properties of the participants' interactivity. We introduce the necessary weights (Role, Hypermedia Package Type, Type of Tool, Type of Service) with a default value equal to one (for zero values, the default is zero). In turn, there is a possibility to modify these values through the Modify option weighting, selecting the coefficient and the coefficient subtype (see Figure 4).

Then, a file is automatically created. This lists all the values needed for the process transfer function tables and the values of their fields that serve as inputs to the DEVS simulator.

\section{Step 3: Setting the metric coefficients}

When it is first accessed to the database, the application generates a file input.csv in which the vectors are saved (eight components) (Rodríguez, Sartorio, \& San Martín, 2010) to be input data environment used to run the DEVS model that integrates the metrics described in section five.

D. Step 4: Running the simulation

Once the correct input values are generated, these will be used in the simulation. DEVS model will run as a 
module (model.exe) that has two parameters: the input file generated before (Input.csv) and the number of total events. After the execution, the output.csv file is generated. This file contains the values of the levels of interactivity for each entry.

\section{E. Step 5: Results}

Figure 5 shows an example of the results obtained from each level of interactivity through time participation in the collaborative environment SAKAI. The interface enables filtering and coloring of various levels of interactivity based on users' requirements.

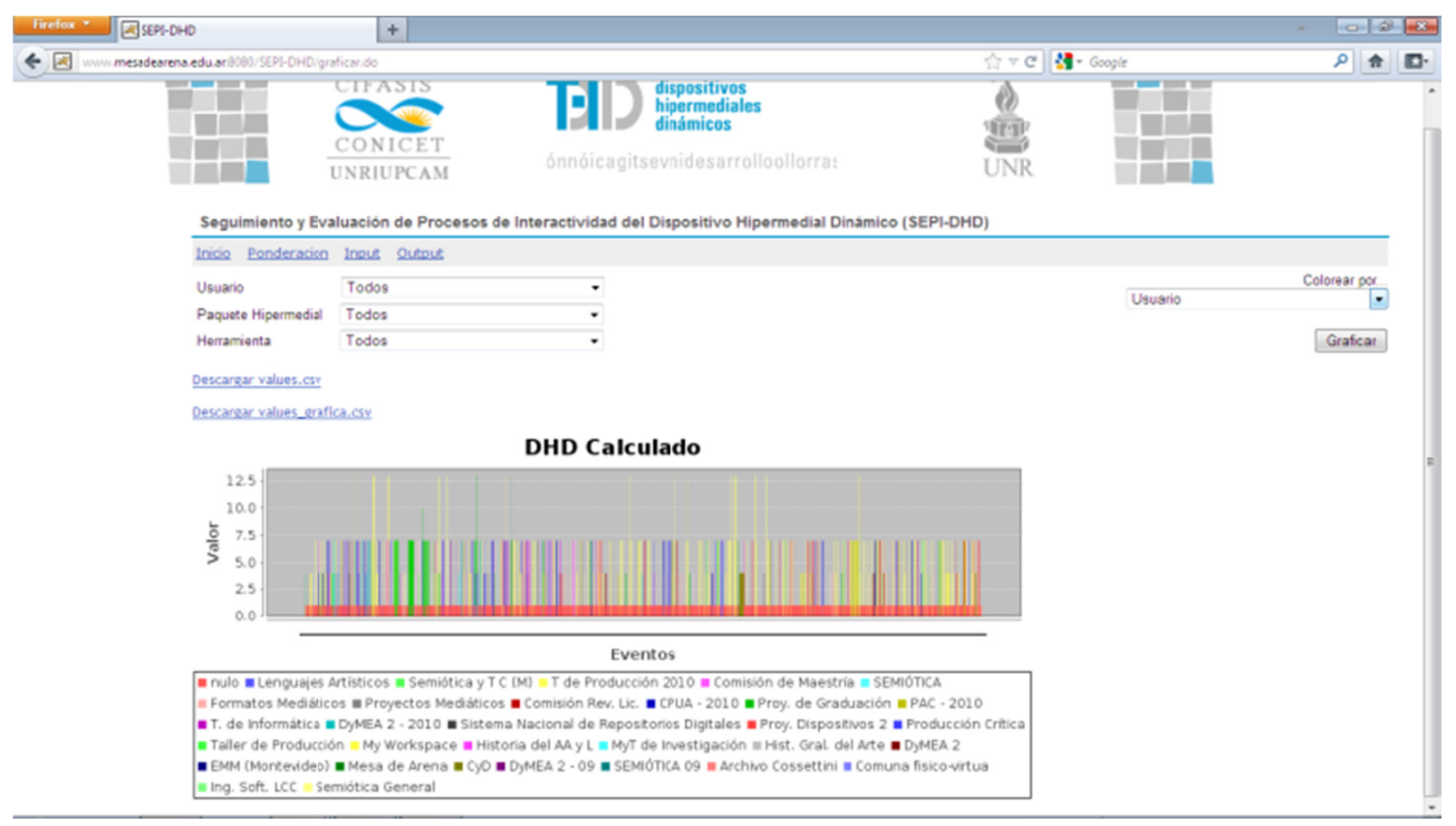

Figure 5. Results obtained by SEPI-DHD

\section{Conclusions}

Based upon DHD systemic modeling, it has been proposed a development and implementation of evaluation metrics for the analysis of the interactivity of Dynamic Hypermedia Device. This analysis is versatile enough to be determined by teachers, researchers, and/or coordinators of the DHD. Thus, it provides reliable information on how to develop processes of responsible participation by socio-technical networks to educate, investigate, manage, and/or produce.

We have presented the design, development and implementation of "SEPI-DHD", which is an integrated tool for Monitoring and Evaluation DHD-Interactivity, adapted to the educational platform MOODLE and the SAKAI collaborative environment. This utility resolves the technological reading of the database, the entry of the coefficients, the implementation of DEVS module that integrates the metrics and presents the results.

As prospective work, the models with appropriate adjustments for the implementation of different application areas (Education, Research, Linking Technology, and Management) should be tested. In turn, it would be necessary to make a development of metrics for monitoring processes in the DHD, taking into account the modeling of the systemic behavior, allowing versatility on 'what' should the application be and 'how' the application meets the requirements and implementations (through a logical design in the domain of the solutions).

To conclude, the DHD invites us to think the plot of inseparable links that constitute the current physical-virtual context. This perspective articulates n-dimensional outlined in the physical materiality of the vast field that involves the body, actions and objects at a time and place set for the virtual area in the dimensions of thought, action, speeches and devices. Our work so far, although not enough in relation to the proposal, intends to enable new insights and questions in terms of promoting better practices for knowledge construction. 


\section{References}

Dujmovic, J., \& Bazucan, A. (1997). A Quantitative Method for Software Evaluation and its Application in Evaluating Windowed Environments. IASTED Software Engineering Conference, Australia.

Gell-Mann, M. (1995). The Quark and the Jaguar. New York: W. H. Freeman.

Guarnieri, G. (2010). El Modo Interactivo del Dispositivo Hipermedial Dinámico. Doctoral Thesis, Universidad Nacional de Rosario. Retrieved from http://rephip.unr.edu.ar/bitstream/handle/2133/1741/Tesis\%20Guarnieri\%20Version\%20Final2.pdf?sequenc $\mathrm{e}=6$. Accessed 25 June 2012.

Lapadula, M., Pagliero, E., Kofman, E., Bergero, F., Namias, R., \& Migoni, G. (2010) Powerdevs, Integrated Tool for Edition and Simulation of Discrete Event Systems (Version 2.0) [Software] Universidad Nacional de Rosario, Argentina: Laboratory for System Dynamics and Signal Processing.

Olsina, L., \& Martín, M. (2004). Ontology for Software Metrics and Indicators. Journal of Web Engineering, 2(4), 262-281.

Olsina, L., Molina, H., \& Papa, F. (2005). Organization-Oriented Measurement and Evaluation Framework for Software and Web Engineering Projects, Lecture Notes in Computer Science of Springer, LNCS 3579, International Congress on Web Engineering, ICWE05.

Olsina, L., \& Rossi, G. (2002). Measuring Web Application Quality with WebQEM. IEEE Multimedia Magazine 9(4), 20-29. http://dx.doi.org/10.1109/MMUL.2002.1041945

Rivera, M. B., Molina, H., \& Olsina, L. (2007). Sistema Colaborativo de Revisión para el soporte de información de contexto en el marco C-INCAMI, XIII Congreso Argentino de Ciencias de la Computación, CACIC 2007. Corrientes y Resistencia, Argentina.

Rodríguez, G. (2010). Desarrollo e implementación de métricas para el análisis de las interacciones del Dispositivo Hipermedial Dinámico, Jornadas Argentinas de Informática, JAIIO 2010. Buenos Aires, Argentina.

Rodríguez, G., \& San Martín, P. (2010). Approximation to the Dynamic Hypermedia Device modeling for the analysis of interactions.Journal of Computer Science and Technology, 10(3), 123-129.

Rodríguez, G., Sartorio, A., \& San Martín, P. (2010). SEPI: una herramienta para el Seguimiento y Evaluación de Procesos Interactivos del DHD, XVI Congreso Argentino de Ciencias de la Computación, CACIC 2010. Morón, Argentina.

Rossi, G., Pastor, O., Schwabe, D., \& Olsina, L. (2007). Web Engineering: Modeling and Implementing Web Applications. Human-Computer Interaction Series. Springer.

San Martín, P., Guarnieri, G., Rodríguez, G., Bongiovani, P., \& Sartorio, A. (2010). El Dispositivo Hipermedial Dinámico - Campus Virtual UNR. Rosario. Secretaría de Tecnologías educativas y de gestión, Universidad Nacional de Rosario.

Sartorio, A., \& Cristiá, M. (2009). First Approximation to DHD Design and Implementation. Clei Electronic Journal, 12(3), Paper $\mathrm{N}^{\mathrm{o}} 1$.

Sartorio, A., \& Rodríguez, G. (2010). Condicionales DEVS en la coordinación de contratos sensibles al contexto para los DHD, XVI Congreso Argentino de Ciencias de la Computación, CACIC 2010. Morón, Argentina.

Zeigler, B. (1976). Theory of modeling and Simulation. New York: John Wiley \& Sons.

Zeigler, B., King, T. G., \& Praehofer, H. (2000). Theory of modeling and Simulation (2nd ed.). New York: Academic Press.

\section{Copyrights}

Copyright for this articleis retained by the author(s), with first publication rights granted to the journal.

This is an open-access article distributed under the terms and conditions of the Creative Commons Attribution license (http://creativecommons.org/licenses/by/3.0/). 